\title{
Brain Hypothermic Therapy Dramatically Decreases Elevated Blood Concentrations of High Mobility Group Box 1 in Neonates with Hypoxic-Ischemic Encephalopathy
}

\author{
Toshihiko Nakamura, ${ }^{1}$ Shingo Yamada, ${ }^{2}$ and Toshirou Yoshioka ${ }^{1}$ \\ ${ }^{1}$ National Hospital Organization, Nishisaitama-Chuo National Hospital, Japan \\ ${ }^{2}$ Central Institute, Shino-Test Corporation, 2-29-14 Oonodai Minami-Ku, Sagamihara-Shi, Kanagawa-Ken 252-0331, Japan \\ Correspondence should be addressed to Shingo Yamada; shingo.yamada@shino-test.co.jp
}

Received 12 June 2013; Accepted 23 August 2013

Academic Editor: Timothy M. Block

Copyright ( $\odot 2013$ Toshihiko Nakamura et al. This is an open access article distributed under the Creative Commons Attribution License, which permits unrestricted use, distribution, and reproduction in any medium, provided the original work is properly cited.

\begin{abstract}
Background. According to the Consensus 2010 of the International Liaison Committee on Resuscitation (ILCOR), children with moderate to severe hypoxic-ischemic encephalopathy (HIE) should receive brain hypothermic therapy (BHT) after successful resuscitation. Elevated high mobility group box 1 (HMGB1) in the blood at the early stage of brain ischemia-reperfusion injury has been suggested to be involved in the release of various inflammatory cytokines. Methods. In total, 21 neonates plasma HMGB1 concentration was measured. These neonates included 8 with HIE in whom BHT was indicated, 5 controls diagnosed as having HIE but who were not suitable candidates for BHT, and 8 normal controls. Results. The umbilical artery HMGB1 (UA-HMGB1) level before undergoing BHT significantly exceeded reference values. The UA-HMGB1 level in the BHT (-) group did not differ significantly from reference values, but was significantly increased 24 hours after birth. Repeated measure ANOVA showed a significant difference in time course changes between the BHT $(+)$ and BHT $(-)$ groups $(P=0.0002)$. Conclusions. This study demonstrated hypothermic therapy to significantly decrease HMGB1. Furthermore, HMGB1 is a useful index of the inhibition of early stage inflammation.
\end{abstract}

\section{Introduction}

High mobility group box 1 (HMGB1) protein was first described almost 35 years ago as a nonhistone chromosomal protein with high electrophoretic mobility [1]. As a DNA binding protein, HMGB1 is involved in maintenance of nucleosome structure and regulation of gene transcription. Recently, Wang et al. found that HMGB1 activated an inflammatory response upon release into the extracellular milieu from necrotic cells [2] and activated macrophages with accompanying organ failure $[3,4]$. HMGB1 has been shown to be a dual-nature protein $[5,6]$.

In humans, serum levels of HMGB1 are increased in several inflammatory diseases such as infection, sepsis and septic shock $[7,8]$, acute coronary syndrome $[9,10]$, and disseminated intravascular coagulation [11].
Failure of circulatory dynamics transfer occurs in approximately $10 \%$ of neonates at birth, and these newborns require supportive interventions, such as suction and stimulation. Furthermore, approximately $1 \%$ of such neonates require aggressive resuscitation, and if appropriate treatment is not given, they may die or suffer serious permanent impairments. According to the Consensus 2010 of the International Liaison Committee on Resuscitation (ILCOR), children with moderate to severe hypoxic ischemic encephalopathy (HIE) [12] should receive brain hypothermic therapy (BHT) after successful resuscitation. BHT is performed as a brain protection strategy for neonates with HIE, mainly to prevent secondary cell death after ischemia reperfusion. Elevated high mobility group box 1 (HMGB-1) in the blood at the early stage of brain ischemia-reperfusion injury has been suggested to be involved in the release of various inflammatory cytokines. 
Recently, we established reference blood values for HMGB1 in neonates [13]. We report herein the results of our first study of HMGB1 dynamics in neonates receiving BHT.

\section{Materials and Methods}

2.1. Study Subjects. In total, 21 neonates who were admitted to the neonatal intensive care unit (NICU) of NishisaitamaChuo National Hospital during the 1-year period starting October 18, 2010, when the Consensus 2010 recommendations were published, were enrolled in this study. These neonates included 8 with HIE in whom BHT was indicated, 5 controls diagnosed as having HIE but who were not suitable candidates for BHT, and 8 normal controls (hospitalized for management of mild respiratory insufficiency; none had HIE). This study was approved by the ethics committee of Nishisaitama-Chuo National Hospital. All participants gave written informed consent.

2.2. Samples. Serum which had been collected for the purpose of postbirth management by a primary care physician in charge of each subject was preserved and used. Umbilical cord blood samples were subjected to plasma separation within 3 hours after birth and stored frozen at $-30^{\circ} \mathrm{C}$. All day 5 plasma samples were obtained by drawing blood via the heelcut method into capillary tubes followed by centrifugation at $12000 \mathrm{rpm}$ and stored frozen at $-30^{\circ} \mathrm{C}$.

2.3. HMGB1 Assay System. The plasma HMGB1 concentration was measured by commercial enzyme-linked immunosorbent assay (ELISA) kit (SHINO-TEST, Japan) according to the suppliers' recommendations [9]. The detection limit of HMGB1 was achieved at concentration above $0.2 \mathrm{ng} / \mathrm{mL}$. Serum samples with 5, 10, and $20 \mathrm{ng} / \mathrm{mL}$ HMGB1 protein concentrations were analyzed in 10 replicates for assessment of intra-assay precision. And so, interassay precision was determined by assaying serum samples of 5,10 , and $20 \mathrm{ng} / \mathrm{mL}$ over 5 separate assays. The intra-assay CVs ranged from $2.9 \%$ to $4.9 \%$, and the interassay CVs ranged from $4.8 \%$ to $8.5 \%$. Recovery, calculated from data with $(n=10)$ several concentrations of 5,10 , and $20 \mathrm{ng} / \mathrm{mL}$ of purified human HMGB1 added to pooled serum as the ratio of the observed concentration to the expected concentration multiplied by $100 \%$, ranged from $92 \%$ to $111 \%$ in the HMGB1 ELISA assay.

2.4. Statistical Methods. Two-way ANOVA and the MannWhitney test were performed using StatMate IV. A $P$ value $<0.05$ was considered to indicate a statistically significant difference.

\section{Results}

Table 1 shows a comparison of the clinical manifestations in cases with and without BHT. Gestational age was significantly greater, and 5-minute Apgar scores were significantly lower in the BHT (+) group. Otherwise, there were no significant differences between these two groups.
TABLE 1: Clinical manifestation in cases with and without BHT.

\begin{tabular}{lccc}
\hline & BHT $(-) n=5$ & BHT $(+) n=8$ & $P$ \\
\hline $\begin{array}{l}\text { Gestational age } \\
\text { (weeks) }\end{array}$ & $35.8 \pm 4.9$ & $40.2 \pm 1.2$ & 0.02 \\
Birth weight (g) & $2235 \pm 953$ & $2716 \pm 391$ & NS \\
Ap-Sc $\left(1^{\prime}\right)$ & $4 \pm 1$ & $3 \pm 1$ & NS \\
Ap-Sc $\left(5^{\prime}\right)$ & $8 \pm 1$ & $5 \pm 1$ & 0.006 \\
UA-pH & $7.16 \pm 0.12$ & $7.03 \pm 0.17$ & NS \\
UA-base excess & $-14.8 \pm 4.9$ & $-14.2 \pm 7.9$ & NS \\
(mmol/L) & $76.2 \pm 31.6$ & $89.0 \pm 47.2$ & NS \\
UA-lactate (mg/dL) & & & \\
\hline
\end{tabular}

Data are means \pm standard deviation.

Ap-Sc: Apgar score; UA: umbilical artery; BHT: brain hypothermic therapy.

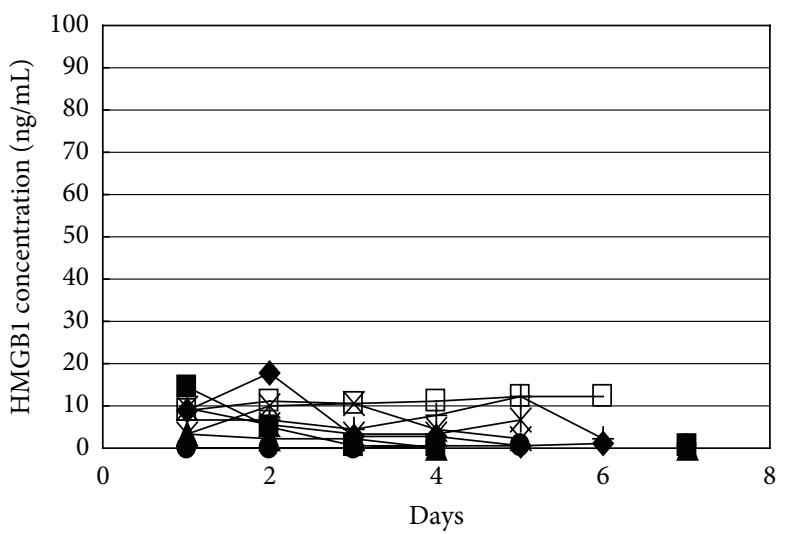

FIgURE 1: Time course in the normal control group. These denote symbols that express 5 controls.

Two-way ANOVA showed significant differences in HMGB-1 levels among cases, but there were no significant differences in individual chronological changes $(P=0.6088)$, indicating that the HMGB1 level did not change significantly from birth through the early stages after birth in normal neonates (Figure 1).

The UA-HMGB1 level before undergoing BHT significantly exceeded reference values. The concentration decreased significantly after BHT, with no further changes through 5 days of age $(P=0.012)$ (Figure 2). The UA-HMGB1 level in the BHT (-) group did not differ significantly from reference values but was significantly increased 24 hours after birth, the time point at which BHT was started in the BHT (+) group, and then was significantly decreased by 5 days of age $(P=0.0102)$ (Figure 2$)$. When values measured at the time point just before the start of BHT were compared, the UA-HMGB-1 level of the BHT (+) group was found to be significantly greater than that of the BHT (-) group. However, the level decreased more significantly in the BHT $(+)$ than in the BHT (-) group 24 hours after the start of this therapy in the former, having no significant difference at 24 hours after birth (Table 2). Repeated measure ANOVA showed a significant difference in chronological changes between the BHT $(+)$ and BHT $(-)$ groups $(P=0.0002)$. 


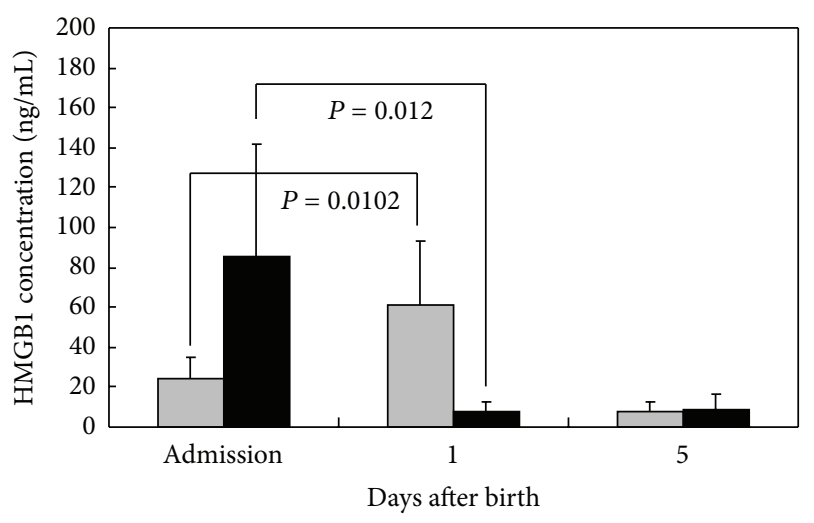

$\square \operatorname{BHT}(-)$
$\operatorname{BHT}(+)$

Figure 2: HMGB1 changes in cases of the BHT (+) and BHT (-) groups. The Mann-Whitney test was performed. These neonates included 8 with HIE in whom BHT was indicated and 5 controls diagnosed as having HIE but who were not suitable candidates for BHT.

TABLE 2: HMGB1 level comparison between the BHT (+) and BHT (-) groups.

\begin{tabular}{lccc}
\hline & $\begin{array}{c}\text { BHT }(-) n=5 \\
(\mathrm{ng} / \mathrm{mL})\end{array}$ & $\begin{array}{c}\text { BHT }(+) n=8 \\
(\mathrm{ng} / \mathrm{mL})\end{array}$ & $P$ \\
\hline Admission & $24.1 \pm 10.8$ & $85.7 \pm 55.8$ & 0.037 \\
1 day after birth & $61.6 \pm 32.4$ & $7.9 \pm 5.1$ & 0.0026 \\
5 days after birth & $7.7 \pm 5.0$ & $8.9 \pm 7.7$ & NS \\
\hline
\end{tabular}

Data are means \pm standard deviation. BHT: brain hypothermic therapy.

\section{Discussion}

There are only a few reports on HMGB1 in neonates. We previously established reference blood values for HMGB1 in full-term neonates soon after birth [13]. There were no significant differences in HMGB1 level related to mode of delivery, but the concentration was significantly increased in cases delivered by emergency cesarean section or vacuum extraction for forced delivery. Furthermore, Okazaki et al. reported the serum HMGB1 level of infants with birth asphyxia to be significantly increased [14].

Studies on the production, secretion, and site of action of HMGB-1 in the body have shown two distinct functions. One involves cell repair and immunoregulatory activity which is a "local HMGB1" function occurring via stimulation due to infection of nuclei such as those of neutrophils and vascular endothelial cells. The other is a "systemic HMGB1" function mediated by HMGB1 secreted by HMGB1 producing cells to induce remote organ failure in the lungs, liver, kidneys, and other organs during hemorrhagic states. The "local HMGB1" and "systemic $\mathrm{HMGB1}$ " functions require approximately 4 hours and 16 hours, respectively $[2,4,5]$.

On the other hand, clinical experiments demonstrated HMGB1 to be released from cells within approximately 1 hour after the onset of brain ischemic lesions, which suggested HMGB1 in the central nervous system to possibly be involved in the early stage of an inflammatory reaction when cytokines such as TNF $\alpha$ and ICAM-1 are produced [15-18].

This study showed the HMGB1 level to already be remarkably increased in the umbilical arteries of infants with birth asphyxia. This result was consistent with those of the abovementioned clinical experiments. Furthermore, neurons were damaged by the sudden onset of asphyxia, one of the ischemic disorders, possibly triggering the rapid release of HMGB1 into the bloodstream after the injury.

BHT or hypothermic therapy for HIE in infants with birth asphyxia is recommended in the Resuscitation Guidelines 2010 Japan. The modes of action of hypothermic therapy vary greatly and include inhibition of brain cellular energy metabolism, inhibition of glutamate release, inhibition of impaired reuptake of glutamate, inhibition of free radical production, reduction of the activities of cytotoxic substances including glutamate, free radicals, and cytokines, and prevention of the metabolic chain reactions characteristic of cellular dysfunction [7-9]. HMGB1 was considered to possibly trigger an inflammatory reaction and thereby promoted the production of cytokines (e.g., TNF $\alpha$ ) which regulate the early inflammatory reaction. This study demonstrated hypothermic therapy to significantly decrease HMGB1. Furthermore, HMGB1 may be a useful index of the inhibition of early stage inflammation.

The sample size in this study was small. Further largescale studies are thus needed.

\section{Disclosure}

The authors have no financial relationships relevant to this paper to disclose.

\section{Conflict of Interests}

The authors have no conflict of interests to declare.

\section{References}

[1] G. H. Goodwin, C. Sanders, and E. W. Johns, "A new group of chromatin associated proteins with a high content of acidic and basic amino acids," European Journal of Biochemistry, vol. 38, no. 1, pp. 14-19, 1973.

[2] H. Wang, O. Bloom, M. Zhang et al., "HMG-1 as a late mediator of endotoxin lethality in mice," Science, vol. 285, no. 5425, pp. 248-251, 1999.

[3] P. Scaffidi, T. Misteli, and M. E. Bianchi, "Release of chromatin protein HMGB1 by necrotic cells triggers inflammation," Nature, vol. 418, no. 6894, pp. 191-191, 2002.

[4] H. Wang, H. Yang, C. J. Czura, A. E. Sama, and K. J. Tracey, "HMBG1 as a late mediator of lethal systemic inflammation," American Journal of Respiratory and Critical Care Medicine, vol. 164, no. 10, pp. 1768-1773, 2001.

[5] H. Wang, H. Yang, and K. J. Tracey, "Extracellular role of HMGB1 in inflammation and sepsis," Journal of Internal Medicine, vol. 255, no. 3, pp. 320-331, 2004. 
[6] H. Wang, H. Yang, C. J. Czura, A. E. Sama, and K. J. Tracey, "HMGB-1 as a late mediator of lethal systemic inflammation," American Journal of Respiratory and Critical Care Medicinel, vol. 164, no. 10, part 1, pp. 1768-1773, 2001.

[7] S. Gaïni, S. S. Pedersen, O. G. Koldkjaer, C. Pedersen, and H. J. Møller, "High mobility group box-1 protein in patients with suspected community-acquired infections and sepsis: a prospective study," Critical Care, vol. 11, no. 2, p. R32, 2007.

[8] J. Sundén-Cullberg, A. Norrby-Teglund, A. Rouhiainen et al., "Persistent elevation of high mobility group box-1 protein (HMGB1) in patients with severe sepsis and septic shock," Critical Care Medicine, vol. 33, no. 3, pp. 564-573, 2005.

[9] S. Yamada, K. Yakabe, J. Ishii, H. Imaizumi, and I. Maruyama, "New high mobility group box 1 assay system," Clinica Chimica Acta, vol. 372, no. 1-2, pp. 173-178, 2006.

[10] R. S. Goldstein, M. Gallowitsch-Puerta, L. Yang et al., "Elevated high-mobility group box 1 levels in patients with cerebral and myocardial ischemia," Shock, vol. 25, no. 6, pp. 571-574, 2006.

[11] T. Hatada, H. Wada, T. Nobori et al., "Plasma concentrations and importance of high mobility group box protein in the prognosis of organ failure in patients with disseminated intravascular coagulation," Thrombosis and Haemostasis, vol. 94, no. 5, pp. 975-979, 2005.

[12] J. J. Volpe, "Hypoxic-ischemic encephalopathy," in Neurology of the Newborn, pp. 245-480, Saunders, Philadelphia, Pa, USA, 2008.

[13] T. Nakamura, S. Yamada, and T. Yoshioka, "Measurement of plasma concentration of high mobility group boxl (HMGB1) in early neonates and evaluation of its usefulness," Clinica Chimica Acta, vol. 413, no. 1-2, pp. 237-239, 2012.

[14] K. Okazaki, M. Kondo, M. Kato et al., "Elevation of highmobility group box 1 concentration in asphyxiated neonates," Neonatology, vol. 94, no. 2, pp. 105-109, 2008.

[15] G. Faraco, S. Fossati, M. E. Bianchi et al., "High mobility group box 1 protein is released by neural cells upon different stresses and worsens ischemic neurodegeneration in vitro and in vivo," Journal of Neurochemistry, vol. 103, no. 2, pp. 590-603, 2007.

[16] J. Qiu, M. Nishimura, Y. Wang et al., "Early release of HMGB1 from neurons after the onset of brain ischemia," Journal of Cerebral Blood Flow and Metabolism, vol. 28, no. 5, pp. 927-938, 2008.

[17] H. Song, Y. Feng, S. Hoeger et al., "High mobility group box 1 and adenosine are both released by endothelial cells during hypothermic preservation," Clinical and Experimental Immunology, vol. 152, no. 2, pp. 311-319, 2008.

[18] A.-G. Ceulemans, T. Zgavc, R. Kooijman, S. Hachimi-Idrissi, S. Sarre, and Y. Michotte, "The dual role of the neuroinflammatory response after ischemic stroke: modulatory effects of hypothermia," Journal of Neuroinflammation, vol. 7, article 74, 2010. 


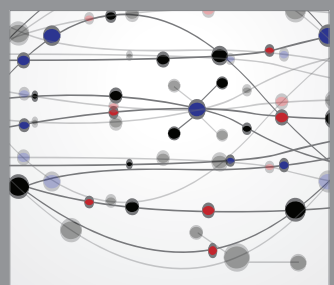

The Scientific World Journal
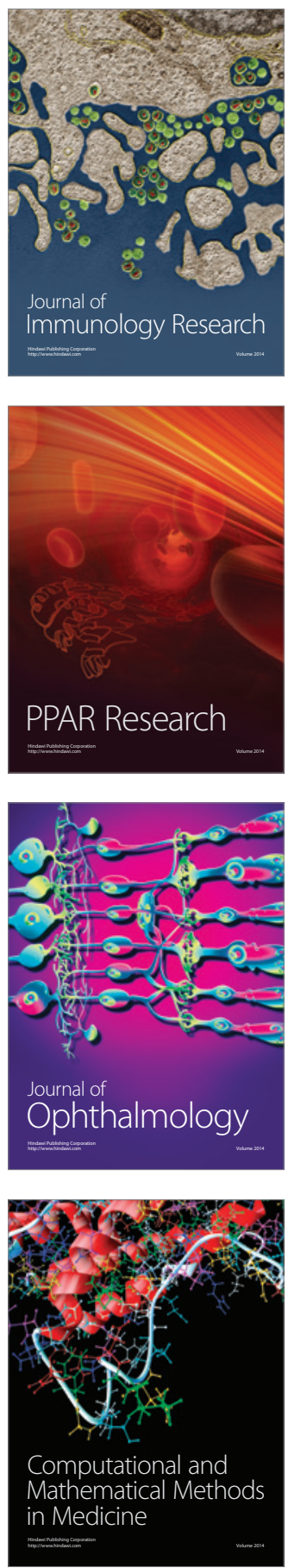

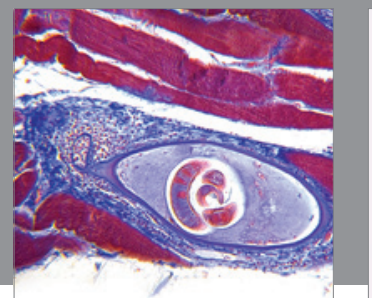

Gastroenterology

Research and Practice
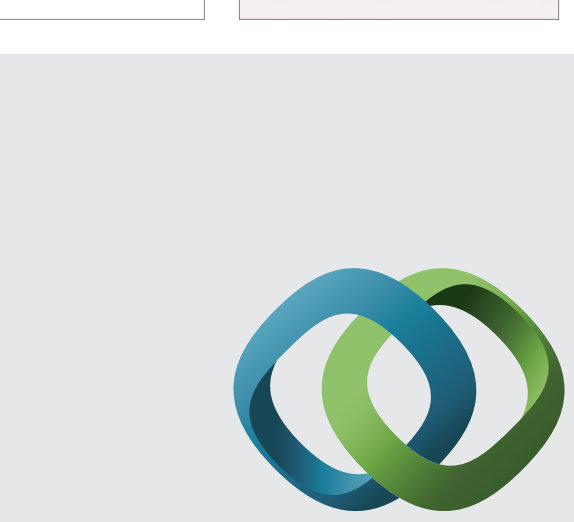

\section{Hindawi}

Submit your manuscripts at

http://www.hindawi.com
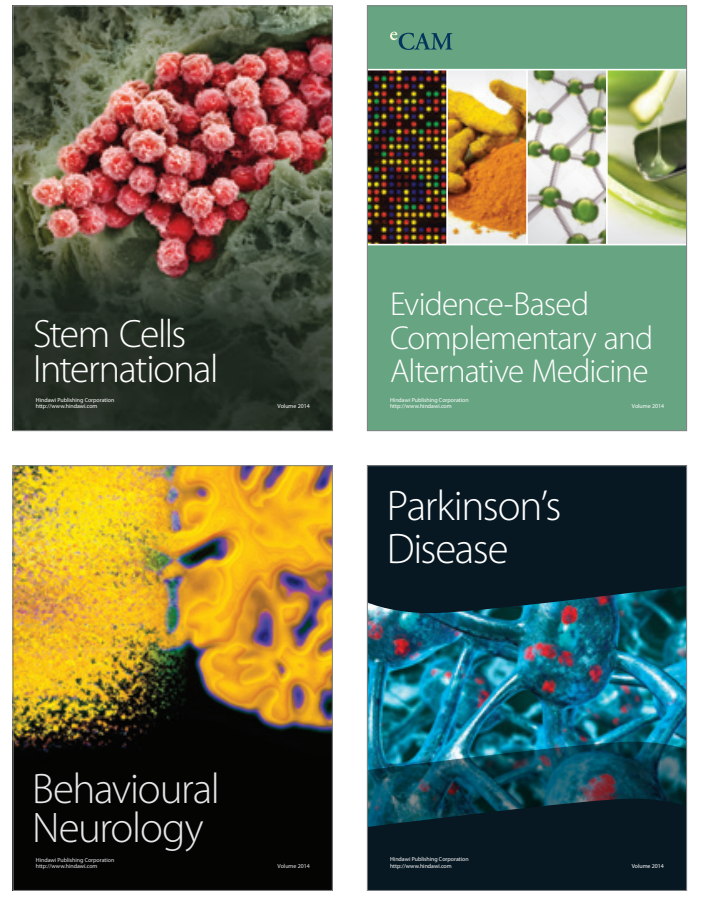
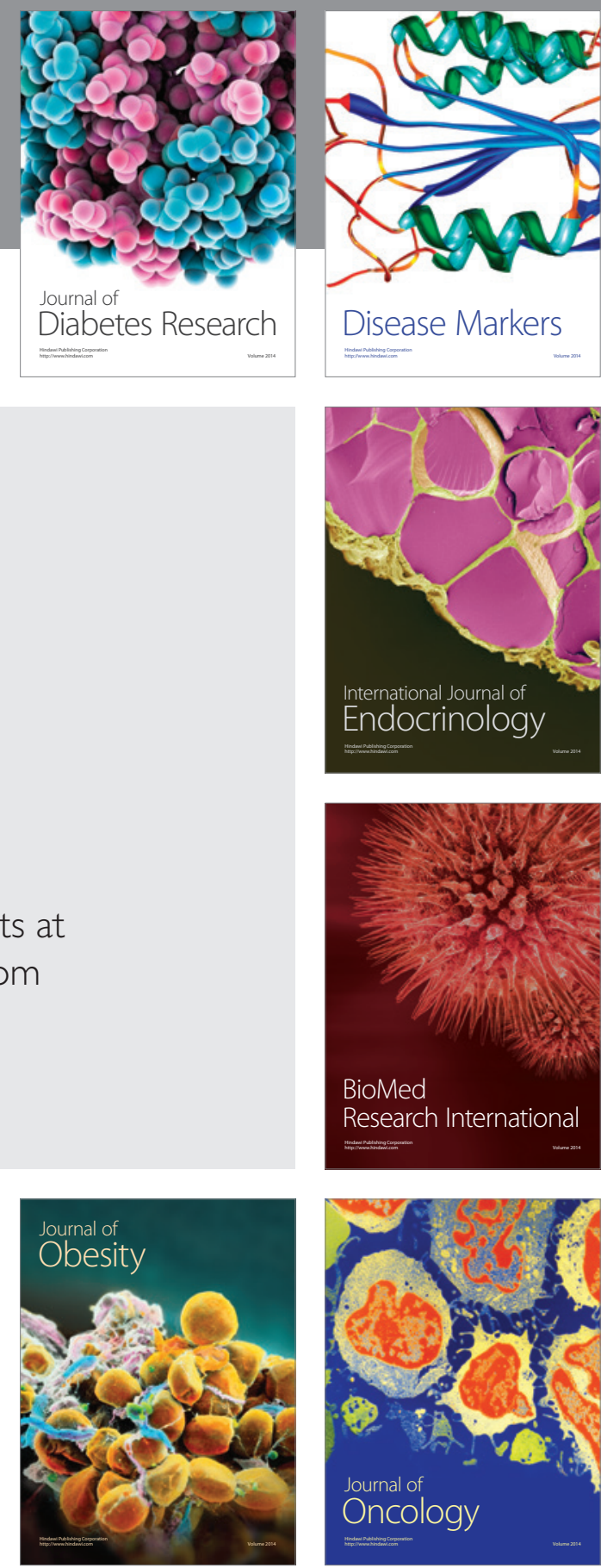

Disease Markers
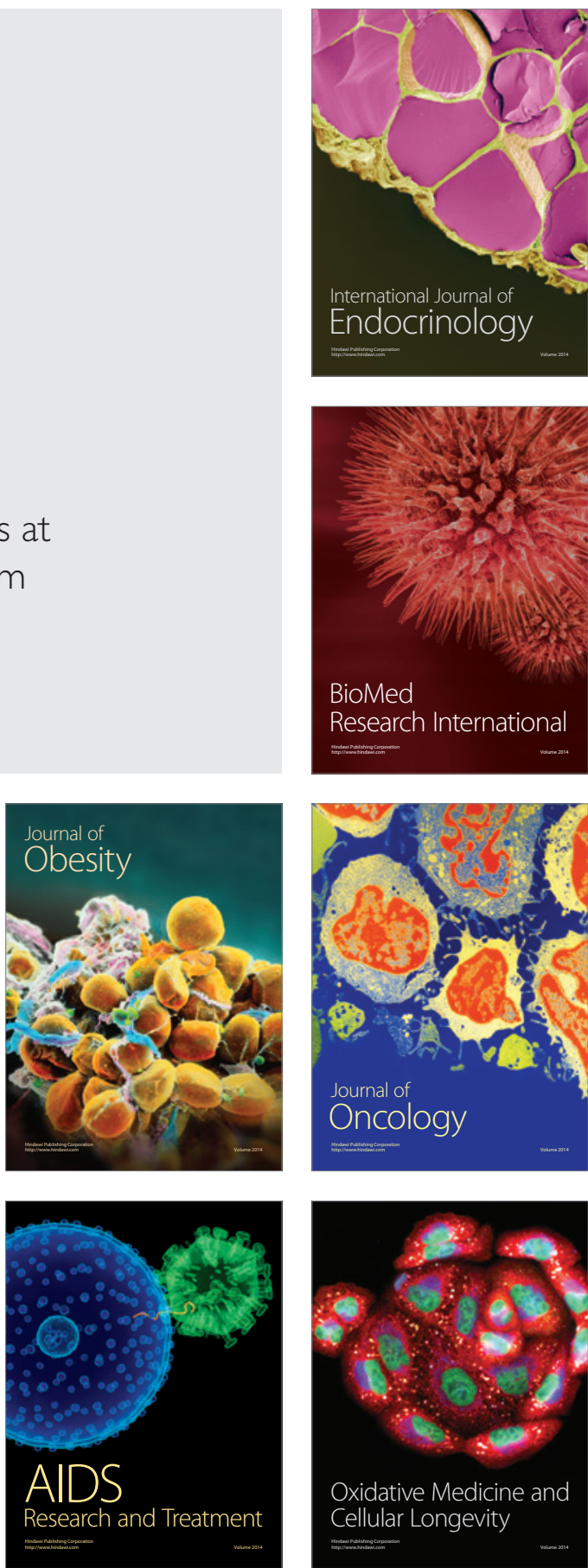